\title{
Oral benfotiamine plus $\alpha$-lipoic acid normalises complication-causing pathways in type 1 diabetes
}

\author{
X. Du • D. Edelstein $\cdot$ M. Brownlee
}

Received: 19 May 2008 / Accepted: 6 June 2008 / Published online: 29 July 2008

(C) Springer-Verlag 2008

\begin{abstract}
Aims/hypothesis We determined whether fixed doses of benfotiamine in combination with slow-release $\alpha$-lipoic acid normalise markers of reactive oxygen species-induced pathways of complications in humans.

Methods Male participants with and without type 1 diabetes were studied in the General Clinical Research Centre of the Albert Einstein College of Medicine. Glycaemic status was assessed by measuring baseline values of three different indicators of hyperglycaemia. Intracellular AGE formation, hexosamine pathway activity and prostacyclin synthase activity were measured initially, and after 2 and 4 weeks of treatment.

Results In the nine participants with type 1 diabetes, treatment had no effect on any of the three indicators used to assess hyperglycaemia. However, treatment with benfotiamine plus $\alpha$-lipoic acid completely normalised increased AGE formation, reduced increased monocyte hexosaminemodified proteins by $40 \%$ and normalised the $70 \%$ decrease in prostacyclin synthase activity from $1,709 \pm 586 \mathrm{pg} / \mathrm{ml}$ 6-keto-prostaglandin $\mathrm{F}_{1 \alpha}$ to $4,696 \pm 533 \mathrm{pg} / \mathrm{ml}$.

Conclusions/interpretation These results show that the previously demonstrated beneficial effects of these agents on complication-causing pathways in rodent models of diabetic complications also occur in humans with type 1 diabetes.
\end{abstract}

\section{Trial registration: NCT00703989}

Funding: Juvenile Diabetes Research Foundation grant 8-2003-784 and GCRC grant MO1-RR12248.

D. Edelstein died during the preparation of this manuscript.

X. Du • D. Edelstein $\cdot$ M. Brownlee $(\bowtie)$

JDRF International Center for Diabetic Complications Research,

Albert Einstein College of Medicine,

Morris Park Avenue, F-531,

Bronx, NY 10461, USA

e-mail: brownlee@aecom.yu.edu
Keywords Advanced glycation endproducts . Benfotiamine $\cdot$ Diabetic complications $\cdot$ Hexosamine pathway $\cdot$ Hyperglycaemia $\cdot$ Lipoic acid $\cdot$ Prostacyclin synthase $\cdot$ Reactive oxygen species $\cdot$ Type 1 diabetes

\begin{abstract}
Abbreviations
PGF prostaglandin $\mathrm{F}$

PKC protein kinase $\mathrm{C}$
\end{abstract}

\section{Introduction}

Benfotiamine blocks three major pathways of hyperglycaemic damage and prevents diabetic retinopathy and incipient nephropathy in experimental models $[1,2]$. In cultured vascular cells, it also reduces aldose reductase gene expression and activity, as well as sorbitol levels [3]. It does so by activating the enzyme transketolase. $\alpha$-Lipoic acid, a potent antioxidant, has also been reported to reduce diabetic microvascular and macrovascular complications in animal models $[4,5]$. To determine whether benfotiamine in combination with $\alpha$-lipoic acid would normalise markers of reactive oxygen species-induced pathways of complications in humans, we performed a pilot study in participants with type 1 diabetes using one daily dose of benfotiamine in combination with $\alpha$-lipoic acid.

\section{Methods}

After protocol approval by the Committee on Clinical Investigations at the Albert Einstein College of Medicine, men with type 1 diabetes and matched healthy controls were recruited. Participant criteria included (in diabetic participants) diabetes duration between 0 and 15 years, current insulin therapy and no evidence of proliferative retinopathy, microalbuminuria, symptomatic diabetic neuropathy or cardiovascular disease. Participants taking any 
medications and those with a history of smoking were excluded. Age of enrolled participants was $28.9 \pm 8.6$ years, duration of diabetes was $25.5 \pm 7.9$ years and BMI was $20.3 \pm 3.1 \mathrm{~kg} / \mathrm{m}^{2}$. All participants gave written informed consent.

The glycaemic status of study patients was assessed by measuring baseline values of $\mathrm{HbA}_{1 \mathrm{c}}$, fructosamine and fasting plasma glucose. Mean $\mathrm{HbA}_{1 \mathrm{c}}$ was $8.7 \pm 0.7 \%$, mean fructosamine was $421 \pm 29 \mu \mathrm{mol} / \mathrm{l}$ (normal range 174$286 \mu \mathrm{mol} / \mathrm{l}$ ) and mean fasting blood glucose was $11 \pm$ $0.49 \mathrm{mmol} / \mathrm{l}$.

At day 0 , levels of markers of two benfotiaminesensitive pathways were determined in participants: (1) intracellular AGE formation, as reflected by a marker of increased intracellular methylgloxal adducts in endothelial cells, angiopoietin 2 [6], and (2) hexosamine pathway activity, measured by determination of $\mathrm{N}$-acetylglucosamine-modified protein in circulating monocytes [7]. Protein kinase $\mathrm{C}$ (PKC) activity in circulating monocytes could not be measured because the amount of blood required exceeded that approved by the Committee on Clinical Investigations. Serum levels of 6-keto-prostaglandin $\mathrm{F}(\mathrm{PGF})_{1 \alpha}$, a stable product produced by the nonenzymatic hydration of the anti-atherogenic mediator prostacyclin, were also determined [8]. Participants then took benfotiamine (300 $\mathrm{mg}$ twice a day; Advanced Orthomolecular Research, Calgary, AB, Canada), together with slow-release $\alpha$-lipoic acid (600 mg twice a day; MRI, San Francisco, CA, USA) for 28 days. Blood was obtained at days 0,15 and 28 .

Data were analysed using one-factor analysis of variance to compare the means of all the groups. The Tukey-Kramer multiple comparisons procedure was used to determine which pairs of means were different.

\section{Results}

An initial study was performed with benfotiamine alone to determine whether the selected dose was sufficient to activate transketolase. In circulating monocytes, the dose of benfotiamine increased transketolase activity by twofold to threefold within 2 weeks $(1.45 \pm 0.17$ vs $3.49 \pm$ $0.22 \mathrm{nmol} \mathrm{min}^{-1}[\mathrm{mg} \text { protein }]^{-1}$, mean $\pm \mathrm{SEM}$ ), an effect similar to that observed in long-term diabetic rat models.

Following this, we examined the effect of combined treatment on angiopoietin-2, a marker of increased intracellular methylgloxal adducts in endothelial cells. Type 1 diabetes was associated with a 1.8-fold increase in circulating angiopoietin-2 levels (Fig. 1a). Treatment with benfotiamine plus $\alpha$-lipoic acid completely normalised angiopoietin-2 levels by 2 weeks $(2,416 \pm 312$ vs $1,062 \pm 176 \mathrm{pg} / \mathrm{ml}$, mean \pm
SEM). Treatment had no effect on any of the three variables used to assess hyperglycaemia in this study (data not shown).

Next, we examined the effect of combined treatment on hexosamine pathway activity by measuring total $\mathrm{N}$-acetylglucosamine-modified proteins in circulating monocytes (Fig. 1b). Type 1 diabetes was associated with a 2.8-fold increase in hexosamine pathway activity $(3,838 \pm$ 765 vs $1,380 \pm 616$ arbitrary units, mean \pm SEM). Two weeks of combined benfotiamine and lipoic acid treatment reduced this value by $40 \%$.

Finally, type 1 diabetes was associated with a $70 \%$ decrease in activity of the critical endothelial anti-atherogenic
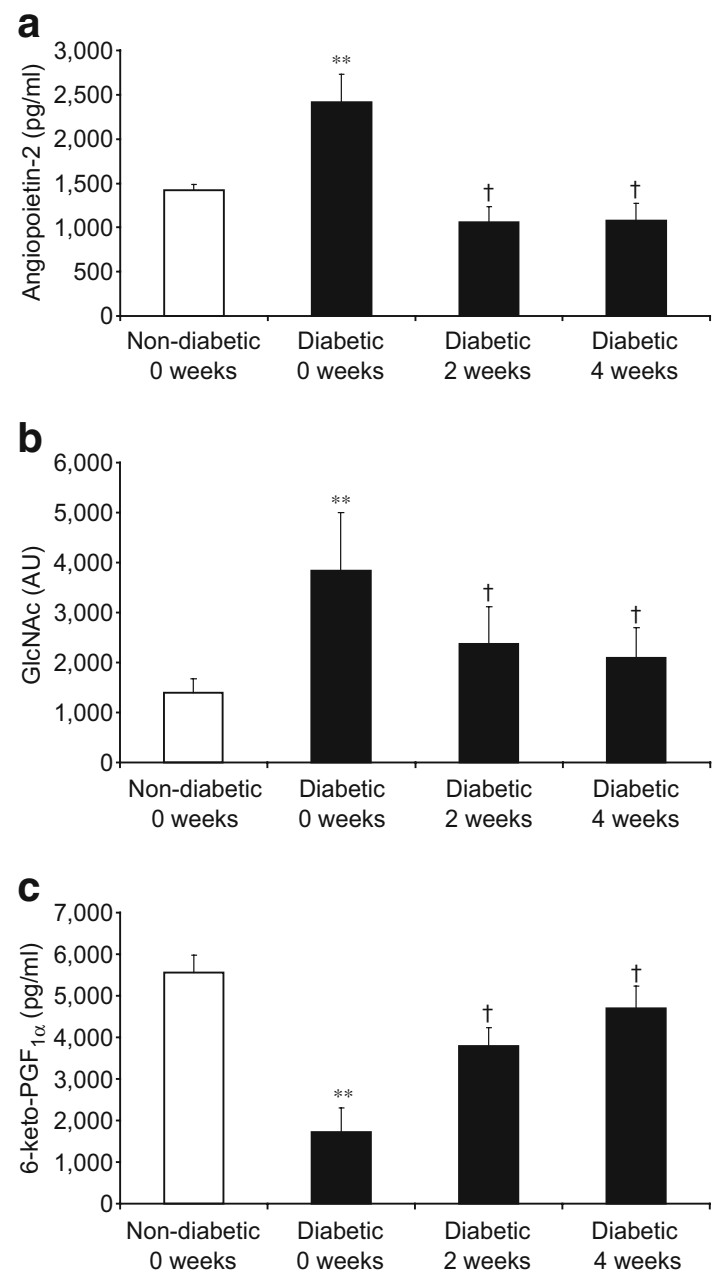

Fig. 1 a Angiopoietin-2 levels in serum of non-diabetic and type 1 diabetic participants before and during treatment with benfotiamine and $\alpha$-lipoic acid. b Intracellular $N$-acetylglucosamine-modified protein (GlcNAc) in monocytes from non-diabetic and type 1 diabetic participants before and during treatment as above (a). c 6-keto-PGF $1 \alpha$ levels in serum of non-diabetic and type 1 diabetic participants before and during same treatment (a). Non-diabetic group $n=12$; type 1 diabetic group $n=9 ; * * p<0.01$ compared with control; $\dagger p<0.01$ compared with week 0 . AU, arbitrary units 
enzyme prostacyclin synthase from $5,775 \pm 294 \mathrm{pg} / \mathrm{ml}$ 6-keto$\mathrm{PGF}_{1 \alpha}$, (mean $\left.\pm \mathrm{SEM}\right)$ to $1,709 \pm 586 \mathrm{pg} / \mathrm{ml}$ 6-keto-PGF ${ }_{1 \alpha}$ (mean \pm SEM) (Fig. 1c). Treatment with benfotiamine plus $\alpha$-lipoic acid normalised 6-keto-PGF ${ }_{1 \alpha}$ activity by 4 weeks $\left(4,696 \pm 533 \mathrm{pg} / \mathrm{ml}\right.$ 6-keto- $\mathrm{PGF}_{1 \alpha}$, mean $\left.\pm \mathrm{SEM}\right)$.

\section{Discussion}

Increased hyperglycaemia-induced superoxide causes glycolytic intermediates to be shunted into the major pathways of hyperglycaemic damage [9]. These intermediates, which activate intracellular AGE formation, the hexosamine pathway and PKC, are also the final products of the transketolase reaction. Because of this, increasing transketolase activity via benfotiamine blocks these complications-causing pathways. Although the damaging pathways inhibited by benfotiamine have been a major focus of complications research, it is important to recognise that excess superoxide can damage vascular cells without involvement of any of these pathways. An important example is the oxidative inactivation of prostacyclin synthase, a critical anti-atherosclerosis endothelial enzyme [10]. For this reason, we combined the antioxidant $\alpha$-lipoic acid with benfotiamine [4].

In this pilot study we report that treatment with oral benfotiamine plus $\alpha$-lipoic acid normalises several complications-causing pathways in patients with type 1 diabetes. The incomplete normalisation of the hexosamine pathway in monocytes, which contrasts with its complete normalisation in rat retina [1], may reflect differential accumulation of benfotiamine in different cell types or a slow turnover of monocyte intracellular proteins. Together, these results show that the beneficial effects of these agents on these pathways, as seen in rodent models of diabetic complications [1], also occur in humans with type 1 diabetes. Replication of these results in a much larger study population after optimisation of benfotiamine and $\alpha$-lipoic acid doses will be necessary in order to determine whether this treatment may help prevent diabetic retinopathy and nephropathy in human patients, as it does in diabetic animal models.

Acknowledgements This work was supported by Juvenile Diabetes Research Foundation grant 8-2003-784 and General Clinical Research Centre grant MO1-RR12248.

Duality of interest The authors declare that there is no duality of interest associated with this manuscript.

\section{References}

1. Hammes H-P, Du X, Edelstein D et al (2003) Benfotiamine blocks three major pathways of hyperglycemic damage and prevents experimental diabetic retinopathy. Nat Med 9:294-299

2. Babaei-Jadidi R, Karachalias N, Ahmed N, Battah S, Thornalley PJ (2003) Prevention of incipient diabetic nephropathy by highdose thiamine and benfotiamine. Diabetes 52:2110-2120

3. Berrone E, Beltramo E, Solimine C, Ape AU, Porta M (2006) Regulation of intracellular glucose and polyol pathway by thiamine and benfotiamine in vascular cells cultured in high glucose. J Biol Chem 281:9307-9313

4. Lin J, Bierhaus A, Bugert P et al (2006) Effect of R-(+)-alphalipoic acid on experimental diabetic retinopathy. Diabetologia 49:1089-1096

5. Yi X, Maeda N (2006) alpha-Lipoic acid prevents the increase in atherosclerosis induced by diabetes in apolipoprotein E-deficient mice fed high-fat/low-cholesterol diet. Diabetes 55:2238-2244

6. Yao D, Taguchi T, Matsumura T et al (2007) High glucose increases angiopoietin-2 transcription in microvascular endothelial cells through methylglyoxal modification of mSin3A. J Biol Chem 282:31038-31045

7. Zachara NE, Hart GW, Cole RN, Gao Y (2002) Detection and analysis of proteins modified by $O$-linked $N$-acetylglucosamine. In: Current protocols in protein science. John Wiley, New York, chapter 17 , unit 17.6

8. Du X, Edelstein D, Obici S, Higham N, Zou M-H, Brownlee M (2006) Insulin resistance reduces arterial prostacyclin synthase and eNOS activities by increasing endothelial fatty acid oxidation. $\mathrm{J}$ Clin Invest 116:1071-1080

9. Brownlee M (2005) The pathobiology of diabetic complications: a unifying mechanism. Diabetes 54:1615-1625

10. Zou MH, Shi C, Cohen RA (2002) High glucose via peroxynitrite causes tyrosine nitration and inactivation of prostacyclin synthase that is associated with thromboxane/prostaglandin $\mathrm{H}(2)$ receptormediated apoptosis and adhesion molecule expression in cultured human aortic endothelial cells. Diabetes 51:198-203 